\title{
RETRACTION
}

\section{Size and topology of exogenous DNA as determinant factors of transgene transcription in mammalian cells}

\author{
H Kamiya, J Yamazaki and H Harashima \\ Graduate School of Pharmaceutical Sciences, Hokkaido University, Sapporo, Japan
}

Gene Therapy (2006) 13, 932. doi:10.1038/sj.gt.3302795

Reference to: Gene Therapy (2002) 9, 1500-1507. doi:10.1038/sj.gt.3301831

In November 2002, Gene Therapy published an article by Professor Hiroyuki Kamiya and colleagues, which described an experiment that sought to construct topology altered DNA from which a transgene product could be efficiently produced.

In December 2005, the authors informed us that they had been unable to reproduce the results of this study and would like to have the paper retracted.

Having considered the authors explanation, which is published below, we have decided to withdraw this paper.

Statement by corresponding author Hiroyuki Kamiya:

Recently, we added a functional DNA sequence to plasmid DNA and examined its effects on transgene expression. At that time, we also linearized the new plasmid DNA, as reported in the Gene Therapy paper. As compared with the circular plasmid DNA, the linearized DNA (DNA dumbbell) did not show the expected, enhanced expression. We checked expression level of the original (without the functional sequence mentioned above) DNA dumbbell and found that the DNA topology had no dramatic effect. We constructed these DNA dumbbells by ligation of oligoDNAs and plasmid DNA digested with restriction enzyme(s). To exclude the possibility that the efficiency of the intermolecular ligation had an influence on the expression, we constructed another plasmid DNA that enables intramolecular ligation. However, the DNA dumbbell prepared from the new plasmid DNA did not increase the expression. Thus, we have to conclude that the DNA dumbbell topology did not enhance transgene expression.

The purpose of our research was to construct topology altered DNA from which a transgene product can be efficiently produced. Although somewhat similar effects were observed by us ${ }^{1}$ and others, ${ }^{2}$ the effects of the topology seem to be highly dependent on the experimental conditions.

We regret that most of the results reported in our Gene Therapy paper are not reproducible and we would like to apologize for this retraction.

\section{References}

1 Tanimoto M, Kamiya H, Minakawa N, Matsuda A, Harashima H. No enhancement of nuclear entry by direct conjugation of a nuclear localization signal peptide to linearized DNA. Bioconj Chem 2003; 14: 1197-1202.

2 van der Aa MA, Koning GA, d'Oliveira C, Oosting RS, Wilschut KJ, Hennink WE et al. An NLS peptide covalently linked to linear DNA does not enhance transfection efficiency of cationic polymer based gene delivery systems. J Gene Med 2005; 7: 208-217. 